\title{
Moving Multiple Dipole Model for Cardiac Activity
}

\author{
Yoshiwo OKamoto, M.A., Yasuaki Teramachi, Ph.D., \\ Toshimitsu Musha, Ph.D., Hiroshi Tsunakawa, M.D., * \\ and Ken-ichi Harumi, M.D.*
}

\section{Summary}

A single-dipole model and a two-dipole model have been examined to approximate the electrical activity of heart; positions as well as vector components of these dipoles were estimated from the body surface potential distribution that was measured with 64 electrodes arranged on the chest. The "residue" has been defined as a measure for how much potential component is left that cannot be attributed to the equivalent dipoles. A locus of the vector end of an equivalent dipole in the single-dipole model is very much like ordinary vectorcardiogram (VCG). The residue has a peak in the last half of QRS; this means that the single-dipole approximation is not valid there. Then another dipole is introduced, which is the two-diple approximation. The residue has been greatly reduced and the peak disappears; the resultant two dipoles move around in the right and left parts of the heart with nearly opposite directions. The moving-two-dipole model for normal subjects describes the cardiac activity in QRS much better than with the moving-single-dipole model.

\section{Additional Indexing Words:}

Models for cardiac activity Excitation fronts Residue

\begin{abstract}
A concept of a moving dipole was first introduced by Gabour and Nelson in 1954.1' Geselowitz proposed a single dipole model and defined an electrical center by a point where a contribution of a quadruple component of a body surface potential distribution is minimized. ${ }^{2), 3)}$ This single moving dipole has been investigated extensively by Brody et al with hearts of isolated turtle $^{4)}$ and rabbit ${ }^{5)}$ suspended in an electrolytic chamber or by Savard et al ${ }^{6)}$ with intact dogs.
\end{abstract}

From the Department of Applied Electronics, Tokyo Institute of Technology, Nagatsuta, Midoriku, Yokohama 227, Japan.

* Division of Cardiology, Showa University Fujigaoka Hospital, Fujigaoka, Midori-ku, Yokohama.

Received for publication August 17, 1981. 
However, this single moving dipole model is sometimes inadequate especially when abnormal hearts are studied. In addition to this, the multipole expansion of the body surface potential distribution needs complicated mathematics and calculation sometimes become unstable. Furthermore, it is remote from intuitive understanding about shapes and locations of the excitation fronts. Thus the estimation of a moving dipole through the multipole expansion has technical difficulties.

Multiple dipole models have been also adopted by several authors. In the model which was proposed by Barber and Fischmann, ${ }^{71}$ the ventricular muscle is divided into a number of segments where each segment has its own dipole which is located at the center of the segment. Lynn et a ${ }^{8)}$ used 12 dipoles which have fixed directions and positions and which always generate currents in outward directions. Since dipole locations are fixed in either model, only linear operations are needed to estimate these dipoles; these models are, however, somewhat poor in representing propagation processes of the excitation fronts.

We employed a multiple moving dipole model and introduced equivalent cardiac dipoles which best simulate electric activity of the heart in the sence of the least-square error. These dipoles would represent locations, directions and sizes of the excitation fronts that generate electric dipole layers.

\section{Methods}

\section{Potential measurement}

A new instrument has been developed for measurement as well as display of the body surface potentials. ${ }^{91,10)}$ The measurement was performed in the following steps.

The 64 electrodes of $\mathrm{Ag}-\mathrm{AgCl}$ disks with diameter $10 \mathrm{~mm}$ are arranged on a chest in a square matrix form of 8 columns and 8 rows. The electrode interval is set to be $4 \mathrm{~cm}$, so that the potentials in a square region of $28 \mathrm{~cm} \times$ $28 \mathrm{~cm}$ are measured.

The potentials with reference to Wilson's central terminal are applied to 64-channel ECG amplifiers and then multiplexed. These signals are converted into 8-bit digital data. Each potential distribution is thus sampled at every $2 \mathrm{msec}$ and stored on 64-kbyte RAM (random access memory). The data on the 64-kbyte RAM are transferred onto a flexible disk with capacity of 256-kbyte. In this process, the offset values of the measured potentials are removed and a drift of the base line is eliminated.

2. Estimation of the equivalent dipoles

An outline of the method for estimating the equivalent dipoles will be 
described and the detailed mathematical explanations will be given in the Appendix.

We first define a proximity function for the n-dipole approximation as a squared deviation of the measured potential distribution from the calculated potential distribution which is generated by $\mathrm{n}$ dipoles $\boldsymbol{p}_{1}, \ldots, \boldsymbol{p}_{\mathrm{n}}$ located at $\boldsymbol{r}_{1}, \ldots, \boldsymbol{r}_{\mathrm{n}}$ inside the thorax. Then, equivalent dipoles are defined as the dipoles which minimize the proximity function. Throughout the analysis an infinitely large, uniform conductor bounded by a plane is assumed as a simplified torso model.

As each dipole has six degrees of freedom (three vector components and three coordinates), the proximity function has $6 \mathrm{n}$ variables. Since vector components are automatically derived from the dipole positions and observed body surface potentials, the independently controlled variables are $3 \mathrm{n}$ dipole positions $\boldsymbol{r}_{1}, \ldots, \boldsymbol{r}_{\mathbf{n}}$. Minimization of the proximity function starts with a trial set of multiple dipole positions; they are moved a step in the so-called steepest descent direction, along which the proximity function decreases most rapidly. This process is repeated until the proximity function reaches a stationary value.

Unless the initial trial dipole positions are pathological, the steepest descent method finds a true minimum of the proximity function. It is not hundred-percent sure, however, that a minimum we get is a true minimum, in other words, that we get the best dipole positions. We have to repeat the whole calculation with another set of initial dipole positions if we get unreasonable result, which has rarely happened.

A minimized root-mean-square deviation between the measured potential distribution and that generated by the equivalent dipoles shall be called "residue" when normalized to the root-mean-square value of the measured potential distribution. That is

$$
\text { Residue }(T)=\sqrt{\frac{S_{\min }(T)}{\sum_{1}{ }^{64} V_{\text {meas }, ~}{ }^{2}(T)}}
$$

where $v_{\text {meas, }}(T)$ is the measured potential at the $\mathrm{i}$-th electrode site at time $T$, and $S_{\min }(T)$ is the minimized root-mean-square deviation. The value of the residue suggests how much potential component is left which cannot be attributed to the equivalent dipoles.

The residue may reflect the complexity of the excitation fronts. If the residue is small with a single dipole, it can be said that the excitation front propagating in the heart has a simple shape. If the residue is large, contrary to this, with a single dipole, there may be plural excitation fronts or the excitation front is largely distorted. In such a case, another equivalent dipole is 
added. If the residue is considerably reduced with two dipoles, it follows there exist two significant excitation fronts, each equivalent dipole representing a mean position, direction and size of the corresponding excitation front. If the residue remains large even with two dipoles, there may be more than two excitation fronts or the excitation front may have a complicated shape.

The root-mean-square (RMS) value of the measured potential represents the electrical activity of the heart. It is defined as

$$
\operatorname{RMS}(\mathrm{T})=\sqrt{{ }_{64}^{1}} \sum_{1^{64} \mathrm{~V}_{\text {meas }, \mathrm{i}^{2}}(\mathrm{~T})}
$$

\section{RESULTS}

Fig. 1 shows the residues and RMS values obtained from 5 normal subjects. Residue in the single- and two-dipole approximations are also plotted with narrow and broad lines. Horizontal axies indicate time with scales of 40 msec.

When the RMS is small, or electrical activity of the heart is low, for example during the early part of QRS, the potential distributions are largely disturbed by noise, which results in a large residue regardless of the type of approximation. It should be noted that each RMS curve has a dip in the last half of QRS, and the corresponding residue curve has a peak there in the single-dipole approximation. When two-dipole approximation is used, this peak disappears and the residue is halved during the last half of QRS except the case (e). Consequently, it can be said that there is only one excitation front in the initial half of QRS and that two excitation fronts exist in the last half of QRS. There are two possibilities to account for this observation. A small excitation front grows larger and larger, splits into two, and they propagate in the opposite directions to each other. Or an excitation front reaches the boundary of myocardium and a hole is made on the excitation front (what is called the break-through). Since the hole can be replaced by an excitation front with a reversed polarization, an excitation front with a hole is expressed as a superposition of two dipole layers, one in the another with a reversed polarization. In either case, body surface potential caused by one of the two excitation fronts is partly killed by the other, so that the RMS decreases and the residue with two dipoles becomes smaller than that of a single dipole.

This consideration is applicable to (c) in Fig. 1, whereas (e) shows a different behavior. Fig. 2 shows again the residue and RMS of the subject (c). The trajectory of the dipole motion in the single-dipole approximation which has been projected on a horizontal plane is shown in Fig. 3; the lower 


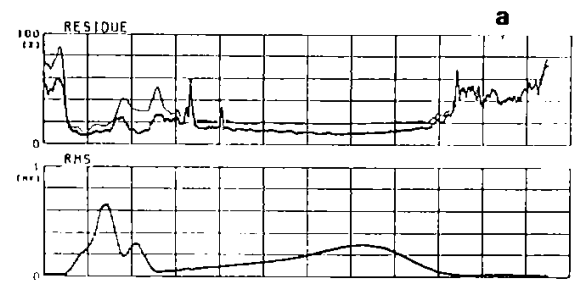

\section{Residue}
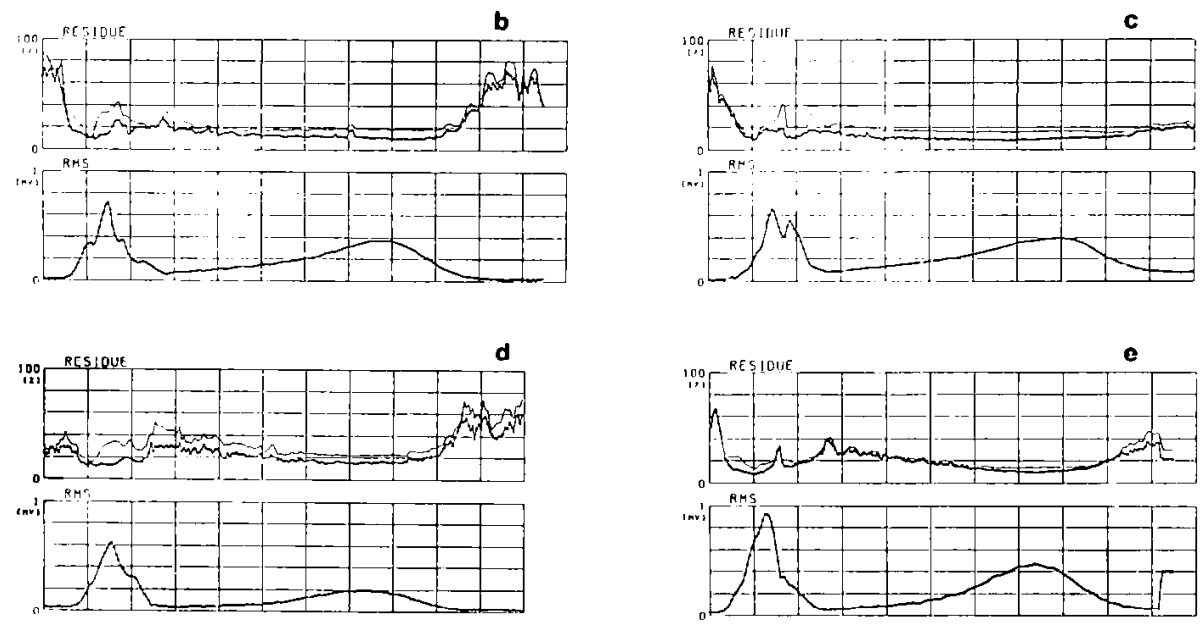

Fig. 1. Residues and RMS values obtained from 5 normal subjects. Residues on the single- and two-dipole approximation are plotted with narrow and broad lines. Horizontal axies indicate time with scales $40 \mathrm{msec}$.

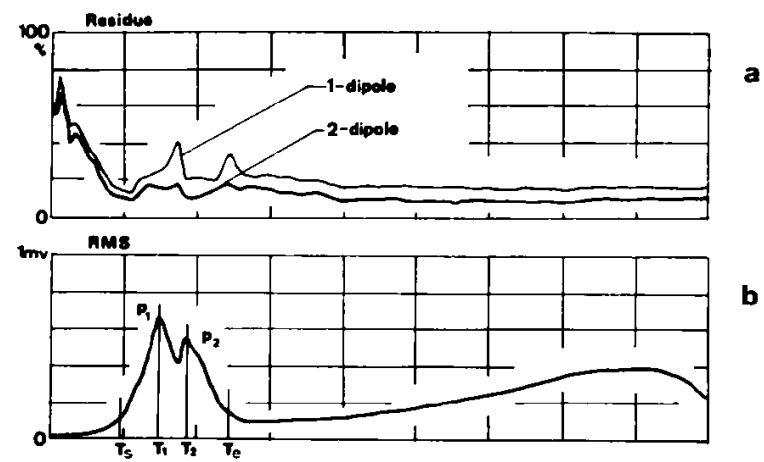

Fig. 2. Residue and RMS curves in the case (c).

edge corresponds to the front chest and a unit length is equal to an electrode distance, $4 \mathrm{~cm}$. The dipole starts at $\mathrm{T}_{s}$ which is indicated in Fig. 2(b) with the same symbol, and the arrow indicates the direction of motion. Dipole positions at every $2 \mathrm{msec}$ (sampling time) are plotted with small dots and successive dots are connected with solid lines. 


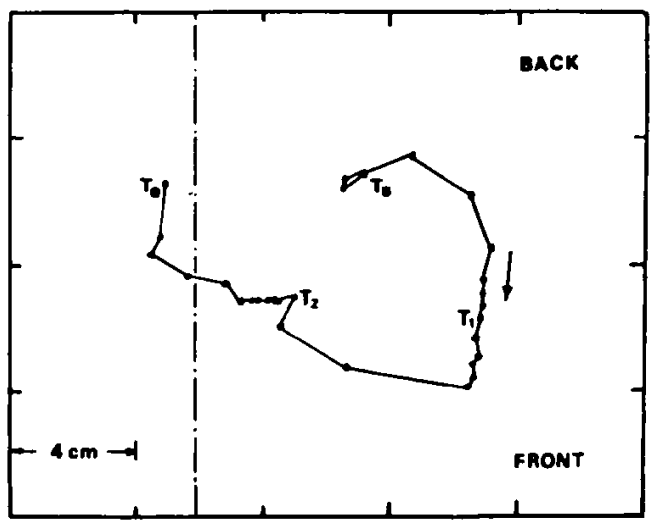

Fig. 3. Trajectory of the equivalent dipole position obtained from singledipole approximation. (Horizontal view: case (c))

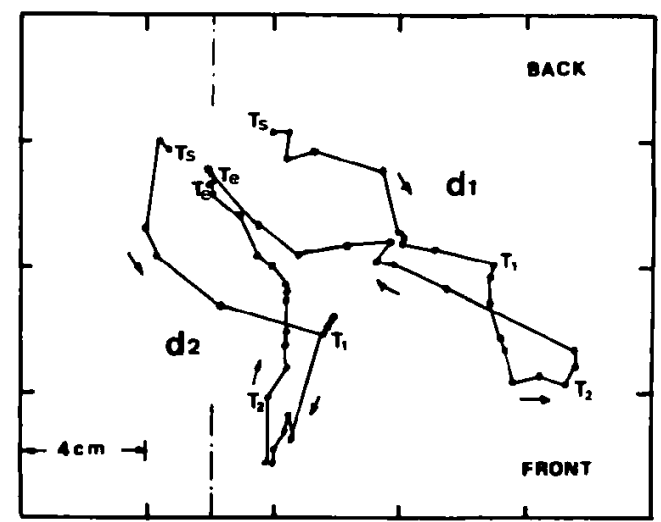

Fig. 4. Trajectories of the two equivalent dipole positions obtained from two-dipole approximation. (Horizontal view: case (c))
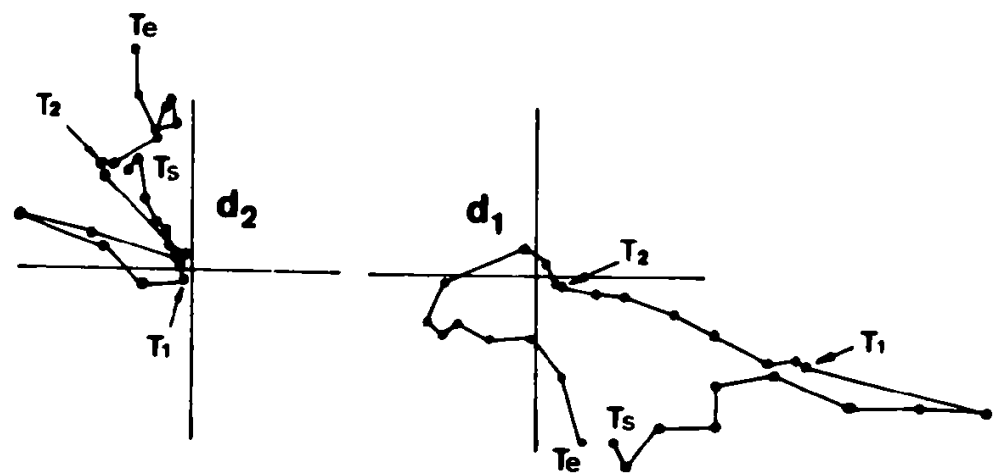

Fig. 5. Vector components of the two equivalent dipoles obtained from two-dipole approximation, in the same fashion as an ordinary vectorcardiogram. (Horizontal view: case (c)) 
Figs. 4 and 5 show positions and vector components of the two equivalent dipoles in the two dipole approximation, which also have been projected on a horizontal plane. Dipoles $d_{1}$ and $d_{2}$ move around in the left and right parts of the heart, respectively. At time $T_{1}$, when the RMS has the peak $p_{1}$ (see Fig. 2(b)), the magnitude of the dipole $d_{1}$ is greater than that of $d_{2}$. Contrary to this, this relation is reversed at time $T_{2}$ where the RMS has the peak $p_{2}$. Thus $d_{1}$ and $d_{2}$ represent the depolarization processes in the left an right ventricles, and the peaks $p_{1}$ and $p_{2}$ occur in the left and right ventricles.

Fig. 6 through 9 show the results referring to the subject (e). Figs. 8 and 9 show positions and moments of the equivalent dipoles in the two-dipole approximation. As in the case (c), two dipoles $d_{1}$ and $d_{2}$ also move in the left and right parts of the heart, but the magnitude of $d_{1}$ is greater than that of $d_{2}$ throughout QRS, actually so great that the trajectory of $d_{1}$ is almost the same as that of the dipole which is obtained from the single-dipole approxi-

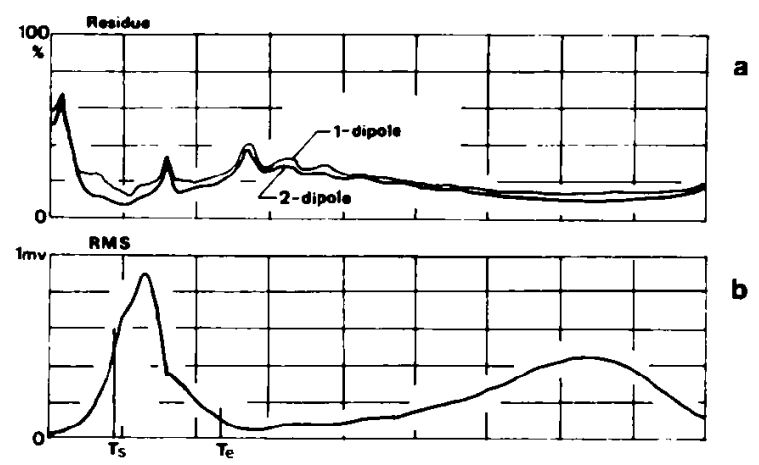

Fig. 6. Residues and RMS values of the potential distribution. (case (e))

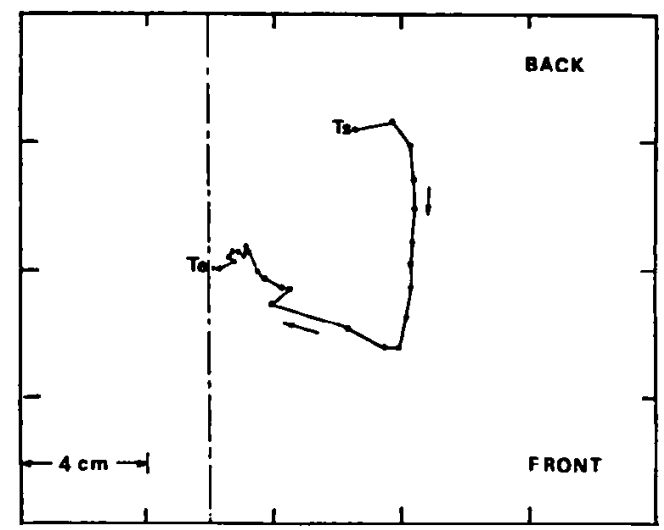

Fig. 7. Trajectory of the equivalent dipole position in the single-dipole approximation. (Horizontal view: case (e)) 


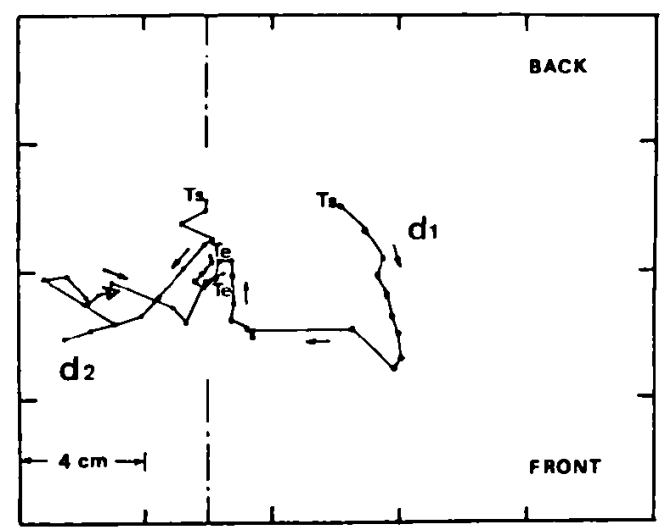

Fig. 8. Trajectories of the two equivalent dipole positions in the twodipole approximation. (Horizontal view: case (e))

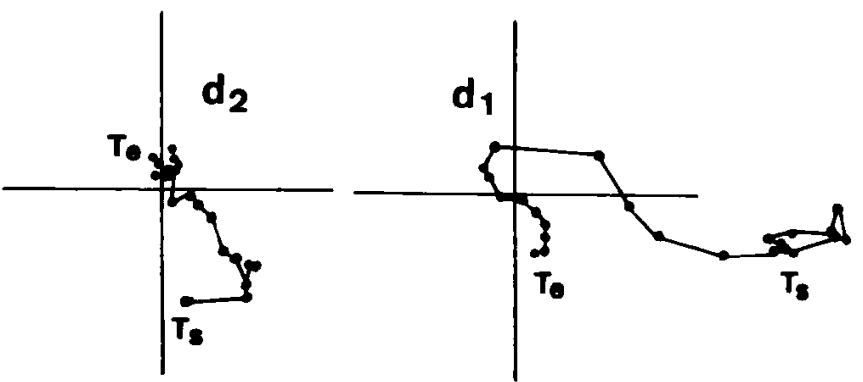

Fig. 9. Vector components of the two equivalent dipoles in the twodipole approximation. (Horizontal view: case (e))

mation. Therefore, it may be said that there exist only one dominant excitation front and several small ones, and that the peak of the residue curve is caused by the break-through. Moreover, it should be noted that the width of the peak of the residue curve in the case (e) is much narrower than that in the case (c). This fact might be also an evidence that the residue peak in the case (e) is caused by the break-through.

\section{Discussion}

The residue obtained from single- and two-dipole approximations have been shown. It seems that more dipoles would yield a better result or reduce a residue. However, calculations with more dipoles become unstable and the results have no longer any practical meaning as explained briefly in the following. Detailed explanations and discussions have been given in reference. ${ }^{11)}$

The relationship between vector components of a dipole located at $\boldsymbol{r}$ in 
the thorax and the body surface potential distribution generated by that dipole can be represented by a transfer matrix $A(r)$ (see Appendix). Suppose that the transfer matrices $\mathrm{A}\left(\boldsymbol{r}_{\mathrm{a}}\right)$ and $\mathrm{A}\left(\boldsymbol{r}_{\mathrm{b}}\right)$ defined at two dipole positions $\boldsymbol{r}_{\mathrm{a}}$ and $\boldsymbol{r}_{\mathrm{b}}$ take almost the same value; then a movement of the dipole from $\boldsymbol{r}_{\mathrm{a}}$ to $\boldsymbol{r}_{\mathrm{b}}$ does not give any appreciable change to the body surface potential distribution as long as the vector components are preserved. Under this condition, it is practically impossible to discriminate between these two dipoles from the body surface potential distribution. With three or more dipoles, rearrangement of the dipole positions gives rise to a small change in the body surface potential, and hence determination of the dipole positions from the body surface potentials becomes difficult or unstable in the presence of noise.

If the potentials on the back, which have not be measured in the present observation, are taken into account, the degree of independence among transfer matrices will be increased and the stability of the calculation will be improved; nevertheless, the three-dipole approximation would be still unstable. It is true that the accuracy of the equivalent dipole estimation will be improved if the true torso model is used at the expence of the CPU time in determining the transfer matrix, but the stability of the calculation may not be improved.

Given a number of degrees of freedom, the moving-multiple-dipole approximation gives a better result than the fixed-multiple-dipole approximation, because the calculation trying to find more dipole is more unstable regardless of whether dipoles have given positions or they are allowed to move.

Some useful information is obtained even with a single moving dipole. For example, a position of the excitation front, which could not be obtained from vectorcardiogram (VCG), can be traced. In particular, the singledipole model is suitable for finding where the excitation front starts from, which is a valuable information in the case of WPW syndrome, since the smaller the excitation front, the more accurate the estimation of equivalent dipoles as long as the signal-to-noise ratio in the measured potentials on the body surface is large enough. The residue provides useful information, because it reflects the complexity of the excitation fronts. For example, when an excitation front reaches an infarction in the myocardium where the electric dipole moment is not developed, a hole is made on a dipole layer of the excitation front and the residue becomes large there. Hence, it is possible to estimate the location and size of the infarction from the timing and the magnitude of a peak in the residue curve. 


\section{Conclusion}

It is found that the two-dipole approximation is very advantageous as compared with the single-dipole approximation especially when excitation fronts in the right and left parts of the heart have comparable magnitudes. By tracing the positions of the two dipoles, we can estimate how excitation fronts are separated and propagate in the ventricles. Residue curve in the single-dipole approximation has a peak during the last half of QRS and this can be attributed to the separation of the excitation front or the break-through.

\section{Appendix}

Calculation of the multiple equivalent cardiac dipoles from the body surface potential distribution

a) Proximity function

Suppose there are $\mathrm{m}$ electrodes arranged on the body surface, and $\mathrm{n}$ equivalent cardiac dipoles located at $\mathrm{n}$ sites are estimated from body surface potentials. A measured potential on the $\mathrm{i}$-th electrode is denoted by $V_{\text {meas, }}$; then distribution of the body surface potential is described as an m-dimensional column vector

$$
V_{\text {meas }}=\left(V_{\text {meas, }, 1, \ldots,} V_{\text {meas,m }}\right)^{t}
$$

In the same way, potentials on these electrode sites that would be generated by the assumed cardiac dipoles $\boldsymbol{p}_{1}, \ldots, \boldsymbol{p}_{\mathrm{n}}$ located at $\boldsymbol{r}_{1}, \ldots, \boldsymbol{r}_{\mathrm{n}}$ are calculated as a column vector as

$$
V_{c a l}=\left(V_{c a l, 1}, \ldots, V_{c a l, m}\right)^{\imath} \text {. }
$$

The squared deviation is then defined by

$$
\mathrm{S}=\left(\mathrm{V}_{\text {meas }}-\mathrm{V}_{\mathrm{cal}}\right)^{\mathrm{t}}\left(\mathrm{V}_{\text {meas }}-\mathrm{V}_{\mathrm{cal}}\right)
$$

The equivalent cardiac dipoles that best approximate the electrical activity of the heart would minimize $S$. As $\mathrm{V}_{\mathrm{cal}}$ is linear in the dipole vector components, one can write

$$
\mathrm{V}_{\mathrm{cal}}=\mathrm{A}\left(\boldsymbol{r}_{1}, \ldots, \boldsymbol{r}_{\mathrm{n}}\right) \mathrm{P} \text {, }
$$

where $A$ is an ( $m: 3 n$ )-transfer matrix and $P$ is a $3 n$-dimensional column vector that consists of the dipole components. This transfer matrix A depends not only on the dipole positions but also on the geometry and the conductance distribution of the thorax, which is assumed unchanged in time. The dipole components are proportional to the body surface potentials and $S$ is minimized with $P_{\text {optm }}$ which is given by

$$
P_{\text {opt } m}=\left(A^{t} A\right)^{-1} A^{t} V_{\text {meas }}
$$

for an arbitrarily given dipole positions. Substituting eq. (A-4) and eq. (A-5) into eq. (A-3), one obtains

$$
S^{\prime}=V_{\text {meas }}^{t}\left(E_{m}-A\left(A^{t} A\right)^{-1} A^{t}\right) V_{\text {meas }},
$$

where $E_{m}$ is an m-dimensional unit matrix. Since $V_{\text {meas }}{ }^{t} V_{\text {meas }}$ is a scalar that is constant for a given potential distribution, one can maximize the second term in the parentheses or the proximity function

$$
\mathrm{I}\left(\boldsymbol{r}_{1}, \ldots, \boldsymbol{r}_{\mathrm{n}}\right)=\mathrm{Vt}_{\text {meas }} \mathrm{A}\left(\mathrm{A}^{\mathrm{t} A}\right)^{-1} \mathrm{~A}^{\mathrm{t}} \mathrm{V}_{\text {meas }}
$$


instead of minimizing $\mathbf{S}^{\prime}$.

b) Transfer matrix

The transfer matrix for $\mathrm{n}$ dipoles can be constructed in terms of the transfer matrix for a single dipole, $\mathrm{A}_{1}(\boldsymbol{r})$;

$$
A\left(r_{1}, \ldots, r_{n}\right)=\left(A_{1}\left(r_{1}\right): A_{1}\left(r_{2}\right): \ldots: A_{1}\left(r_{n}\right)\right)
$$

Here, $A_{1}(r)$ depends, as mentioned above, on the geometry and conductance distribution of the thorax; it would take a long time to evaluate matrix elements for a complexed torso model, and hence the torso model is simplified as a uniform, infinitely large conductor bounded by a plane. Under this assumption, the image method yields

$$
\left[\mathrm{A}_{1}(\boldsymbol{r})\right]_{\mathrm{ij}}=\frac{1}{2 \pi \sigma} \cdot \frac{\left(\boldsymbol{r}-\boldsymbol{r}_{1}\right) \cdot e_{j}}{\left|\boldsymbol{r}-\boldsymbol{r}_{\mathrm{i}}\right|^{3}}, \quad \mathrm{i}=1 \sim \mathrm{m}, \mathrm{j}=\mathrm{x}, \mathrm{y}, \mathrm{z}
$$

where

$\boldsymbol{r}_{1}=\mathrm{i}$-th electrode position on the body surface

$\boldsymbol{r}=$ dipole position in the thorax

$e_{\mathrm{j}}=$ unit vector of the $\mathrm{j}$-th axis

$\sigma=$ electric conductivity.

c) Optimization process

The steepest descent method (SDM) is used to maximize the proximity function. Dipole positions $\boldsymbol{r}_{1}, \ldots, \boldsymbol{r}_{\mathrm{n}}$ can be represented by a vector $\boldsymbol{X}$ in a $3 n$-dimensional space. From an initial position vector $\boldsymbol{X}_{0}$, the final vector is successively approached. In this process, the $(k+1)$-th vector $\boldsymbol{X}_{\mathrm{k}+1}$ is evaluated from the $k$-th vector in the following way. As a first step, unit gradient vector $e_{k}$ of the proximity function at $\boldsymbol{X}_{\mathrm{k}}$ is calculated as

$$
\boldsymbol{e}_{\mathrm{k}}=\operatorname{grad}(\mathrm{I}) /|\operatorname{grad}(\mathrm{I})|_{\mathbf{x}=\mathrm{Xk}} .
$$

Next we define a function $F$ of one variable $g$ by

$$
\mathbf{F}(\mathrm{g})=\mathrm{I}\left(\boldsymbol{X}_{\mathrm{k}}+\mathrm{g} \boldsymbol{e}_{\mathrm{k}}\right)
$$

and find $g_{o p t m}$ which maximize $F$. Then the $(k+1)$-th vector $\boldsymbol{X}_{k+1}$ is determined as

$$
\boldsymbol{X}_{\mathrm{k}+1}=\boldsymbol{X}_{\mathrm{k}}+\mathrm{g}_{\mathrm{optm}} \boldsymbol{e}_{\mathrm{k}} \text {. }
$$

These processes are repeated until the proximity function reaches a stational value.

d) Initial dipole positions

The potential measurement is made at every $2 \mathrm{msec}$. This time interval is so short that the difference between the successive potential distribution is very small. This is shown as follows. A correlation function between a potential distribution at time $T_{i}$ and that at the previous time $T_{i-1}$ is evaluated as
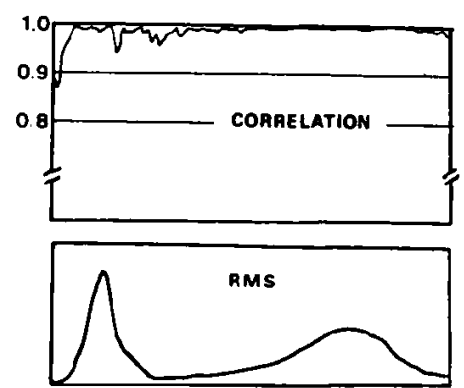

Fig. A-1. Correlation between a potential distribution at a specified time and that at $2 \mathrm{msec}$ before. 


$$
r\left(T_{1}\right)=V_{\text {meas }}^{t}\left(T_{1-1}\right) V_{\text {meas }}\left(T_{i}\right) /\left|V_{\text {meas }}\left(T_{1-1}\right) \| V_{\text {meas }}\left(T_{1}\right)\right|
$$

Fig. A-1 shows how this correlation function varies as time goes on. The correlation exceeds 0.95 through QRS and 0.99 through $\mathbf{T}$; therefore, the final vector $\boldsymbol{X}$ at time $\mathbf{T}_{i-1}$ is taken as the initial vector $\boldsymbol{X}_{0}$ at the next sample time $T_{i}$. This cannot be applicable, however, to the first data. In this case, a number of trial positions are tried with the help of random numbers, and one which yields the maximum proximity function is selected as an initial position.

\section{References}

1. Gabor D, Nelson CV: Determination of the resultant dipole of the heart from measurements on the body surface. J Appl Phys 25: 413, 1954

2. Nelson CV, Gesclowitz DB: The Theoretical Basis of Electrocardiology, Chap 9, Clarendon Press, Oxford, p202, 1976

3. Geselowitz DB: Two theorems concerning the quadrupole appricable to electrocardiography. IEEE Trans Biomed Eng (BME) 12: 164, 1965

4. Brody DA, Warr OS, Wennemark JR, Cox JW, Keller FW, Terry FH: Studies of the equivalent cardiac generator behavior of isolated turtle hearts. Circulat Res 29: 512, 1971

5. Brody DA, Cox JW, Keller FW, Wennemark JR: Dipole ranging in isolated rabbit hearts before and after right bundle branch block. Cardiovasc Res 8: 37, 1974

6. Savard P, Roberge FA, Perry JB, Nadeau RA: Representation of cardiac electrical activity by a moving dipole for normal and ectopic beats in the intact dog. Circulat Res 46: 415, 1980

7. Barber MR, Fischmann EJ: Heart dipole regions and the measurement of dipole moment. Nature (Lond) 192: 141, 1961

8. Lynn M, Barnard ACL, Holt J, Sheffield L: A proposed method for the inverse problem in electrocardiology. Biophys J 7: 925, 1967

9. Kanou $T$, Teramachi Y, Musha $T$, Tsutumi T, Harumi $\mathrm{K}$ : Dynamic display of body surface potential. 17th Conference Japan Soc, ME \& BE 1-c-25, p88, Jul 1978

10. Harumi K, Musha T: Body surface mapping of ECG potentials. Abstructs $I$ of 8 th World Congress of Cardiology, S-27, p57, Sept 1978

11. Okamoto Y, Teramachi Y, Musha T: Instability in the calculation of the equivalent cardiac dipoles. (to be published)

12. Okamoto Y, Teramachi Y, Musha T, Tsutsumi T, Harumi K: Motion of equivalent electric dipole to cardiac activity estimated from body surface potentials. Jpn Heart J 21: 761, 1980 